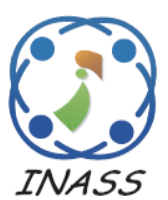

http://www.inass.org/

\title{
Robust Parameter Estimation of an Electric Vehicle Lithium-Ion Battery Using Adaptive Forgetting Factor Recursive Least Squares
}

\author{
Mouncef Elmarghichi $^{1 *} \quad$ Mostafa Bouzi $^{1} \quad$ Naoufl Ettalabi $^{1}$ \\ ${ }^{1}$ Laboratory of Mechanics, Computer science Electronics and Telecommunications \\ Faculty of Sciences and Technology, Hassan first University, Morocco \\ * Corresponding author’s Email: elmarghichi.mouncef@ gmail.com
}

\begin{abstract}
In this paper, a battery model suitable for electric vehicle application is analyzed. Open circuit voltage is described by an adaptation of Nernst equation. Thevenin circuit is used to depict the instantaneous and transient regime. Hysteresis effect is outlined by a zero-state correction term. We propose a new algorithm AFFRLS (adaptive forgetting factor recursive least squares) to extract the parameter of the battery model, then to predict the output voltage, and compare it to the original FFRLS (forgetting factor recursive least squares). To evaluate these algorithms, we used experimental data conducted by CALCE Battery Research Group on the Samsung INR 18650-20R battery cell. We fed the data to the algorithms and compared the estimated output voltage for two dynamic tests on MATLAB. Results show that AFFRLS has low distribution in high error range up to 4\% less than FFRLS, this means that AFFRLS has a better parameter identification than FFRLS.
\end{abstract}

Keywords: Forgetting factor recursive least squares (FFRLS), Adaptive forgetting factor recursive least squares (AFFRLS), Lithium-ion battery, Nernst equation, Electric vehicle (EV).

\section{Introduction}

One of the biggest keys to fighting climate change and urban pollution is to bring electricity to transportation at a large scale. With more electric vehicles on the road, less fuel is burnt and less planetheating gases is put out into the atmosphere.

An electric vehicle relies on a battery to supply the energy to different components of the vehicle. The most appealing and attractive choice for electrical and hybrid electrical vehicles (VE, HEV) are lithium battery.

Battery management system (BMS) ensures good operation of the battery. BMS supervises not only the battery via different indicators ( $\mathrm{SOC}, \mathrm{SOH} . .$.$) , but$ also guarantees the safety and balance between different cells [1].

To help improve the system performance and reliability, and to lengthen the lifetime span of the battery, the battery management system needs to accurately predict the state of charge (SOC), as a matter of fact the SOC estimation is one of the main tasks of a BMS. Precise SOC estimation of the battery can avoid unpredicted system interruption and prevent the batteries from being overcharged and over discharged [1-3], which may cause permanent damage to the internal structure of batteries.

Kalman filters [2-5], Sliding mode observers [6, 7], techniques and others have been used heavily to estimate battery state of charge. The performance of these methods relies heavily on the accuracy of the battery model.

When the battery is used, some parameters in the battery equivalent model change when the working conditions change, affected by the factors such as ambient temperature, operating conditions, and battery aging degree. Therefore, it is necessary to accurately identify these parameters.

In this context various works were carried out, we discuss here the most recent: In [8], parameters of a Thevenin model are identified based on the voltage response data collected from a battery under constant current discharging. From the voltage response, a sensitivity analysis allows to analyze the 
identifiability of the parameters. To address identification, an optimization problem based on nonlinear least squares is formulated, bounds were imposed to limit the search space. The problem of identification is solved by a trust region method. In [9], a third order ECM (equivalent circuit model) is used to describe a lithium battery, the coevolutionary particle swarm optimization (CPSO) method is applied to identify battery parameters. A PIW parameter identification window is used to hold a piece of data that indicates the battery operation at the present moment, and used afterwards by the CPSO to identify the battery parameters. To optimize their values, each parameter employs a separate parameter particle swarm and changes only one step in every cycle. In [10], FFRLS (forgetting factor recursive least squares) is applied to steadily refresh the parameters of a Thevenin model and a nonlinear Kalman filter is used to perform the recursive operation to estimate SOC (state of charge). In [11] an adaptive online estimation algorithm for fractional equivalent circuit model is proposed based on the theory of fractional order calculus and indirect Lyapunov method. In [12], an online parameter identification strategy is proposed to track the parameters deviation guaranteed by Lyapunov's direct method. Unlike other estimation techniques where temperature effects are ignored, the mentioned paper proposes a universal compensation strategy which can be used with many estimation algorithms. In paper [13], a particle filter PF is used to identify in real time the battery model parameters with consideration of the battery states. Meanwhile, a cubature Kalman filter is used to estimate the state of charge SOC.

The above algorithms are accurate, but a part from RLS (recursive least squares), they are computationally expensive (CPSO, PF), this infer that RLS is much faster [14]. Also, the implementation of RLS is easier in the chips which suits electric vehicle application. In addition, some of the mentioned algorithms (PF, CPSO) need a precise model to achieve optimal results, this is not the case for RLS.That's why in this paper we focus on RLS technique method which is commonly used for system parameter identification [14].

The problem with RLS is the fact that it is affected by old data, this means that the generation of new data will be affected by old data, which will lead to large errors. This is adjusted by introducing a forgetting factor into the RLS, so that the proportion of old data is reduced when new data is available, and the algorithm can converge to the actual value quickly [15].
Since the forgetting factor is constant, the ability and accuracy of dynamic circuit parameters identification using FFRLS will be affected when the charging and discharging currents change frequently, especially when the fluctuations are vigorous, and that's the case for electric vehicle application.

In this paper we propose an adaptive expression to calculate the forgetting factor and compares it with the fixed forgetting factor FFRLS applied to the model proposed in [16]. The model is based on the one RC Thevenin equivalent model. Open circuit voltage (OCV) and state of charge (SOC) relationship is described using an adaptation of Nernst equation, the model also uses zero state hysteresis correction term to describe hysteris phenomenon [16].

We will expose the simulation results carried out with MATLAB to verify the performance and accuracy of the AFFRLS versus FFRLS algorithm in two dynamic profiles: Dynamic stress test (DST), and the Federal Urban Driving Schedule (FUDS).

The paper is organized as follows: section 2 describes the model structure used. section 3 reports the principle of the adaptive forgetting factor proposed. section 4 describes the implementation of the proposed on AFFRLS. section 5 reports the simulation setup, results and discussion. Finally, Section 6 presents the conclusion.

\section{Lithium-Ion battery modeling}

The battery model is the same as used in [16]. The model contains one resistance $R 2$ to simulate the instantaneous voltage drop, $R 2$ represents the internal resistance of the battery. One parallel RC network to describe the transient regime $(R l, C l)$. The open circuit voltage $(O C V)$ is described using an adaptation of NERNST equation with three parameters $K 0, K 1$ and $K 2$. sM refers to hysteresis effect, $\mathrm{s}$ is a function of the current sign in the battery. $V$ is the terminal voltage, and $M$ is a coefficient term that needs to be identified.

The equivalent circuit model is shown in Fig. 1. The sign s according to [16]:

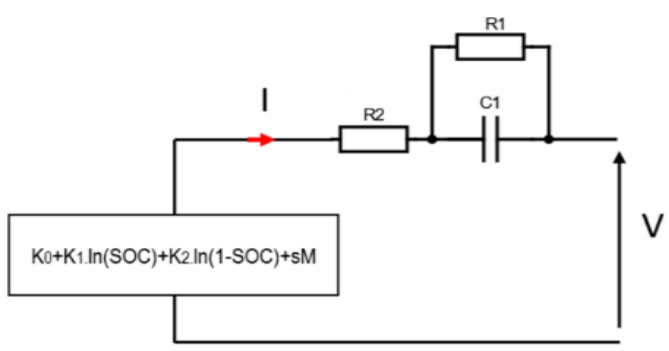

Figure. 1 Battery cell model 


$$
s(k)=\left\{\begin{aligned}
& 1 \text { if } I(k)>\varepsilon \\
&-1 \text { if } I(k)<-\varepsilon \\
& s(k-1) \text { if }|I(k)| \leq \varepsilon
\end{aligned}\right.
$$
time.

$\varepsilon$ is a small positive number and $\mathrm{k}$ being the index

In [16] the following discrete time state equations are used to calibrate recursively the parameters ( $K O$, $K 1, K 2, M, R 2, R 1, C 1)$ using recursive least square with fixed forgetting factor $\lambda$ :

$$
\operatorname{SOC}(k+1)=\operatorname{SOC}(k)-\frac{\eta I(k) \Delta t}{C n}
$$

$\mathrm{Cn}$ is the nominal capacity of the battery, $\eta$ is the Coulombic efficiency which is assumed to be $100 \%$ for discharge and $98 \%$ for charge. $I(k)$ is the current flowing in and out from the battery, $I(k)$ is positive at discharge and negative at charge. $\Delta t$ represents the sampling time interval.

$$
\begin{aligned}
V(k)= & K_{0}(k)+K_{1}(k) \ln (S O C(k))+I(k) R_{2}(k) \\
& +K_{2} \ln (1-S O C(k))+S(k) M(k)-A(k) U_{1}(k-1) \\
& -B(k) I(k-1)
\end{aligned}
$$

$V(k)$ is the terminal voltage in sample time k, with:

$$
\begin{aligned}
& A(k)=\exp \left(\frac{-\Delta t}{R_{I}(k) C_{I}(k)}\right) \\
& B(k)=R_{l}(k)\left[1-\exp \left(\frac{-\Delta t}{R_{l}(k) C_{l}(k)}\right)\right]
\end{aligned}
$$

The parameters to be identified ( $K O, K 1, K 2, M$, $R 2, R 1, C l)$ are grouped in vector $\theta(k)$.

$$
\begin{aligned}
\theta(k)= & {\left[K_{0}(k), K_{1}(k), K_{2}(k), M(k), R_{2}(k), A(k)\right.} \\
& B(k)]^{T}
\end{aligned}
$$

With:

$$
\begin{aligned}
& \varphi(k)=[1, \ln (\operatorname{SOC}(k)), \ln (1-S O C(k)), s(k),-I(k), \\
& \left.-U_{1}(k-1),-I(k-1)\right] \\
& \quad U_{1}(k-1)=K_{0}(k-1)+K_{1}(k-1) \ln (\operatorname{SOC}(k-1))+ \\
& K_{2}(k-1) \ln (1-S O C(k-1))+s(k-1) M(k-1)- \\
& I(k-1) R_{2}(k-1)-U_{1}(k)
\end{aligned}
$$

$U_{l}(k-1)$ represents the previous voltage drop across the resistor $R 1$.

$$
\begin{aligned}
& G(K)=\frac{P(k-1) \varphi(k)}{\lambda+\varphi^{T}(k) P(k-1) \varphi(k)} \\
& P(K)=\frac{P(k-1)-G(k) \varphi^{T}(k) P(k-1)}{\lambda}
\end{aligned}
$$

$G(K), P(k)$ represent the gain and error covariance matrices respectively.

$\lambda$ is the forgetting factor used to give less weight to older data and more weight to recent data.

$$
\theta(k)=\theta(k-1)+G(k)\left[V(k)-\varphi^{T}(k) \theta(k-1)\right]
$$

In [16], fixed weight forgetting factor FFRLS uses the above equation to predict the new parameter values stored in $\theta(k)$ vector, based on the previous values hold on $\theta(k-1)$, the gain matrix $G(K)$ and the error covariance matrix $P(k)$.

The figure below illustrates the schematic diagram of the recursive least square method used to identify the parameters.

\section{Adaptive forgetting factor}

The forgetting factor $\lambda$ assigns weights for old and new data and usually takes a constant value between 0.95 and 1 [13]. $\lambda$ used in [16] is constant.

However, it is expected from the forgetting factor to vary adaptively with the identification parameter error. especially when the online identification parameter error is very large to make the online identification have faster convergence speed and reduce the identification error [15].

The crucial part of the variable forgetting factor least squares algorithm is how to make the forgetting factor adaptively change. An adjusted equation inspired from [15] to calculate the adaptive forgetting factor is proposed to achieve the above goal, it is expressed by:

$$
\begin{aligned}
& \lambda(k)=\min \left(\lambda_{\text {min }}+\left(1-\lambda_{\text {min }}\right) \cdot h^{\varepsilon(k)}, 1\right) \\
& \varepsilon(k)=\frac{e(k)}{e_{\text {base }}}
\end{aligned}
$$

$\lambda_{\min }$ is the minimum value of the forgetting factor fixed at 0.98 to give better balance between accuracy and speed [15].

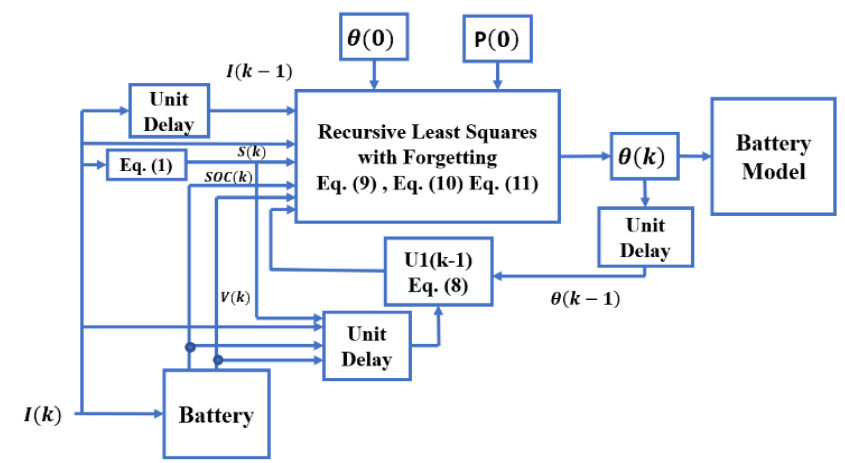

Figure. 2 Schematic of the recursive least square method for battery model update [16] 
$\mathrm{h}$ is a constant coefficient, it indicates the sensitivity of the forgetting factor with respect to errors. $\lambda$ changes slowly when $\mathrm{h}$ is close to 1 and leads to slow response speed. When it is close to 0 itchanges sharply, which speeds up the parameter identification response, but reduces the accuracy. We used the same value 0.9 as in [15].

$e(k)$ is the error at time $\mathrm{k}$, and $e_{\text {base }}$ is chosen based on the magnitude of the expected error.

The identification parameters are considered stable when $e(k)$ error is less than,$\lambda$ in this case changes to a larger value. When the error of identification parameters $e(k)$ is greater than $e_{\text {base }}$, the identification parameters are considered unstable and $\lambda$ changes to a smaller value.

It can be seen from Eq. (12) that the larger the error value $e(k)$, the smaller the forgetting factor, and vice versa, its variation range is between 0.98 and 1 . Thus, the forgetting factor changes with the error of identification parameters.

\section{Implementation of online parameter identification algorithm based on AFFRLS}

The first order RC model equivalent circuit described in section 2 is used as a model where the parameters to be estimated at time $\mathrm{k}$ are hold in vector $\theta(k)$ Eq. (6).

AFFRLS is applied recursively to calculate this vector with adaptive weight factor $\lambda$ reported in section 3 . The specific implementation flow chart of AFFRLS proposed is shown in Fig. 3.

The initial values of the parameter estimate $\theta(0)$, the error covariance matrix $P(0)$, the gain matrix $G(0)$, the sensibility $h$, and forgetting factor $\lambda$, are firstly provided. Then, the $\theta(k)$ parameter vector can be updated based on the online collected regressor.

For every iteration, the previous $U_{k-1}$ is calculated Eq. (8), then SOC (state of charge) is readjusted according to equation Eq. (2).

Before calculating $\theta(k)$, the gain matrix $G(k)$ is assessed according to Eq. (9).

When $\theta(k)$ is evaluated Eq. (11). The estimated values of $R 1, R 2, C 1, K 0, K 1$, and $K 2$ at time k can be obtained.

The covariance matrix $P(k)$ are updated for every iteration by the obtained gain matrix $G(k)$ Eq. (10).

At the end, the estimated voltage can be calculated Eq. (3), and a new value of the forgetting factor $\lambda$ is computed Eq. (12) based on the current error $e(k)$ and the expected error $e_{\text {base }}$.

The parameter identification at the next moment are performed again using the same steps.

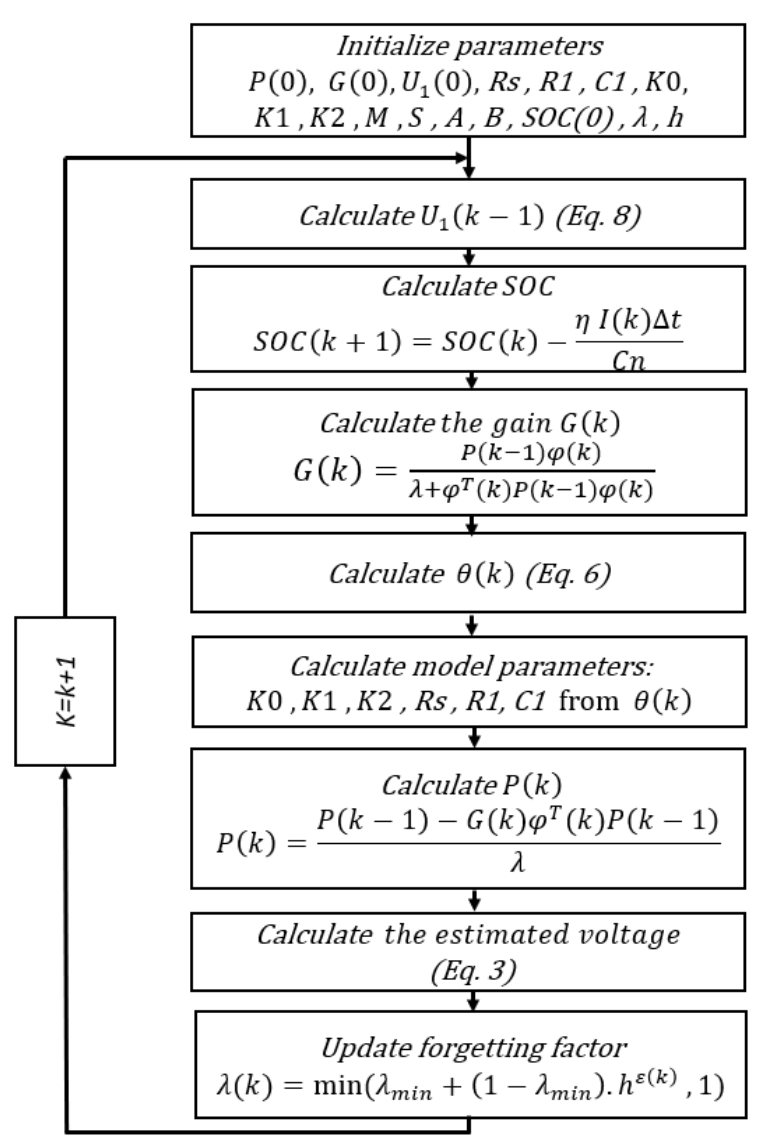

Figure. 3 Flow chart of the adaptive forgetting factor recursive least square proposed AFFRLS

\section{Simulation setup, results and discussion}

In this paper, we propose a new algorithm AFFRLS (adaptive forgetting factor recursive least squares) Fig. 3 to extract the battery parameter and estimate the output voltage. we compare in this section the suggested algorithm (AFFRLS) to the original FFRLS (forgetting factor recursive least squares) Fig. 2 proposed in [16] applied to the same model described in section 2.

To verify the performance of the two algorithms, we used experiment data provided by CALCE Battery Research Group operated on the Samsung (INR 18650-20R) battery cell. This type of lithiumion cell is suitable for EV (electric vehicle) application due to its high voltage and capacity.

We used two datasets provided by CALCE Battery Research Group. The two datasets store the results (current, voltage, time...) of two EV (electric vehicle) cycles conducted on the Samsung (INR 18650-20R) lithium-ion battery named The Dynamic stress test (DST) and the Federal Urban Driving Schedule (FUDS). The table below shows the specifications of this battery [18-20]. 
Table 1. Battery parameter

\begin{tabular}{|c|c|}
\hline \multicolumn{2}{|c|}{ INR 18650-20R Battery cell } \\
\hline Battery (Parameters) & Specifications (Value) \\
\hline Capacity Rating & 2000mAh \\
\hline Cell Chemistry & LNMC/Graphite \\
\hline Max current (A) & 22 \\
\hline Cut-off voltage & $2.4 / 4.2$ \\
\hline
\end{tabular}

The CALCE Battery Research Group used a custom built battery test bench which included lithium battery cells, a thermal chamber to control temperature, an Arbin BT2000 battery test system to control battery charge and discharge, and a host computer with Arbin software to observe and control data information in order to obtain these data [18-20].

The constant current constant voltage (CCCV) was used to charge the Samsung (INR 18650-20R) battery cell. The measurements were recorded in a 1second interval. Data were collected at a low temperature of $0^{\circ} \mathrm{C}$, room temperature of $25^{\circ}$, and a high temperature of $45^{\circ} \mathrm{C}$ [18-20]. We used data that was carried out for $80 \%$ battery level at $25^{\circ} \mathrm{C}$.

We fed the data (current and voltage, time....) to both algorithms applied for the same model discussed in section 2, and compared the estimated terminal voltage for the two dynamic tests (DST and FUDS) with the measured voltage recorded in the datasets.

We discuss the results for DST and FUDS profiles in section 5.1 and 5.2 respectively.

Fig. 4 and Fig. 5 illustrate the current profile for each dynamic test, we can see that these two experiments have a strict charge and discharge process, the cell is highly stressed with a current that varies between $+2 \mathrm{~A}$ (charge) and $-4 \mathrm{~A}$ (discharge).

Underneath these terms, the validity of the parameter identification algorithms can be well verified.

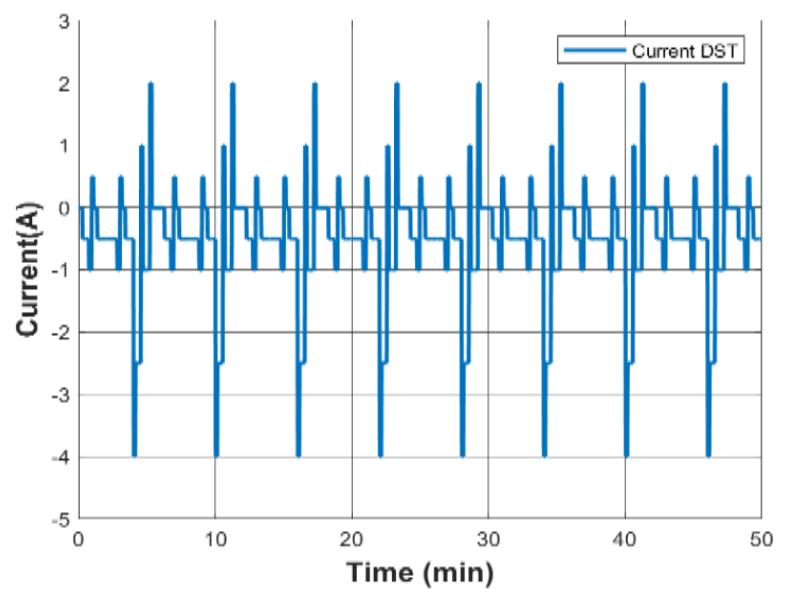

Figure. 4 Current for DST profile at $25^{\circ} \mathrm{C}$

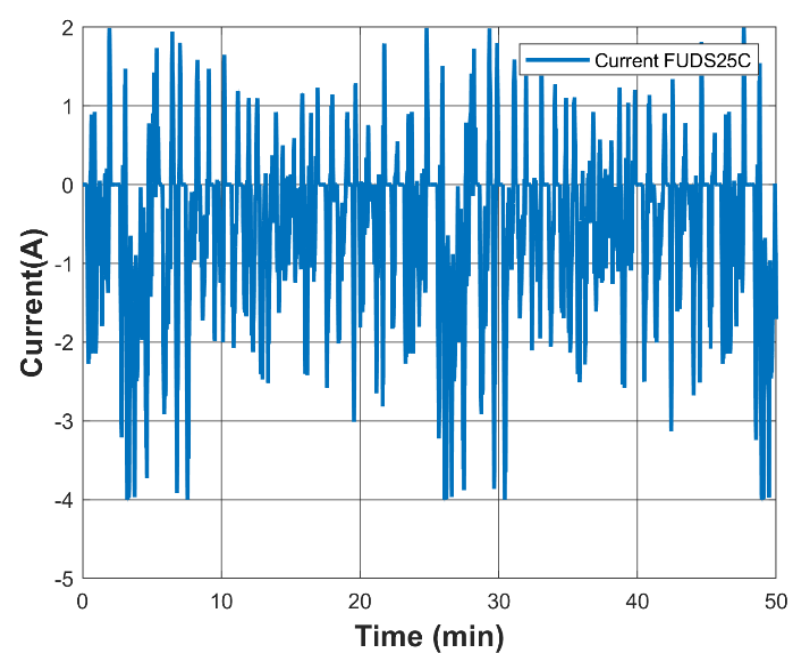

Figure. 5 Current for FUDS profile at $25^{\circ} \mathrm{C}$

\subsection{Dynamic stress test (DST)}

The DST is a common driving cycle which is often used to assess various battery models and SOC (state of charge) estimation algorithms, we used it here to compare the two algorithms. The current profile of this test is shown in Fig. 4.

We provided the same initial values for both AFFRLS and FFRLS: The vector that holds the battery parameters to be estimated $\theta(0)$ Eq. (6). The gain and error covariance matrices $\mathrm{G}(0)$ and $\mathrm{P}(0) \mathrm{Eq}$. (9) Eq. (10) respectively. The initial voltage drops U1 (0) across the resistor R1 Eq. (8). We provided as well forgetting factor $\lambda$ for both algorithms.

We run afterwards in MATLAB the algorithms FFRLS Fig. 2 and AFFRLS Fig. 3 to estimate the battery parameters and the output voltage of the battery.in each iteration we supply the algorithms with the current and voltage stored in the datasets provided by CALCE battery group research for this test. In Fig. 6, we collected the parameter identification results for FFRLS and AFFRLS for DST test. In Fig. 7 we plot the output voltage estimated by both algorithms and compare it with the measured voltage (true value stored in dataset).

It can be seen that the parameters identified by the AFFRLS algorithm are relatively stable. The parameters evolve smoothly with the change of charging and discharging current. In fact, AFFRLS relies on both the forgetting factor $(\lambda)$ which oscillate vigorously in this test Fig. 6, and the parameters stored in $\theta(k)$ Eq. (6) to reduce the error between the estimated and the true terminal voltage. In contrary FFRLS has a fixed $\lambda$, thus, to reduce the error only parameters in vector $\theta(k)$ vary, as a consequence we observe more variations and sometimes spikes in parameters identified by FFRLS. 


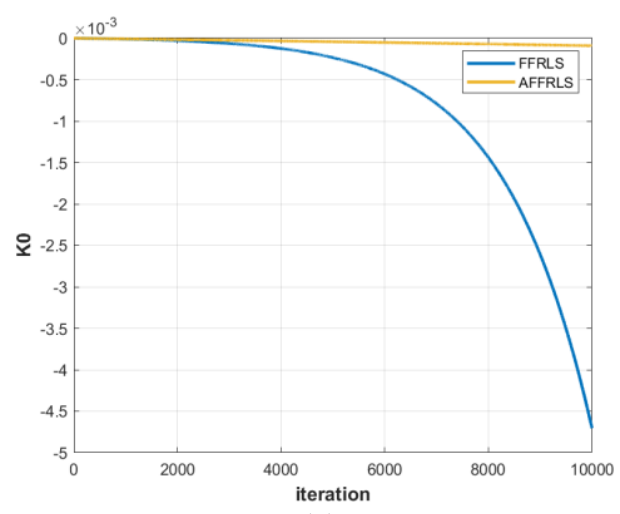

(a)

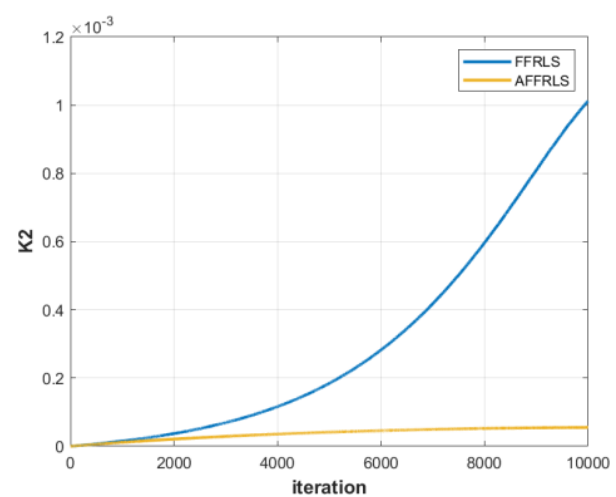

(c)

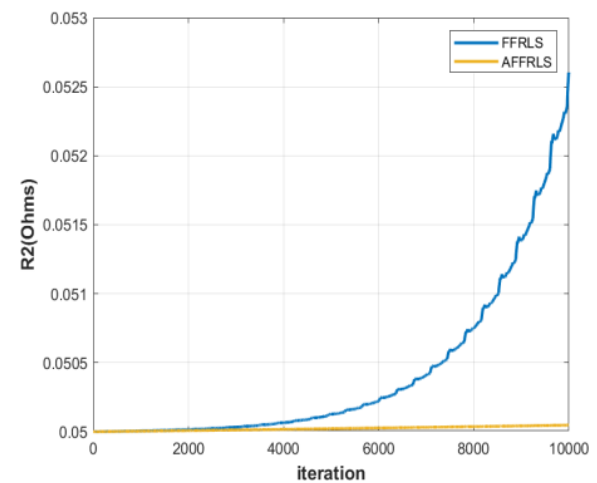

(e)

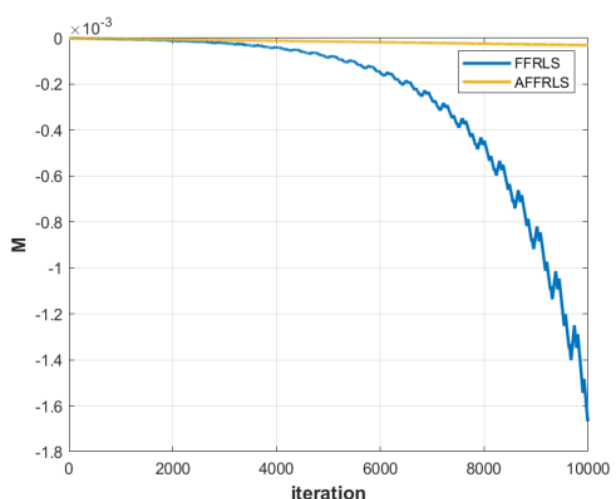

(g)

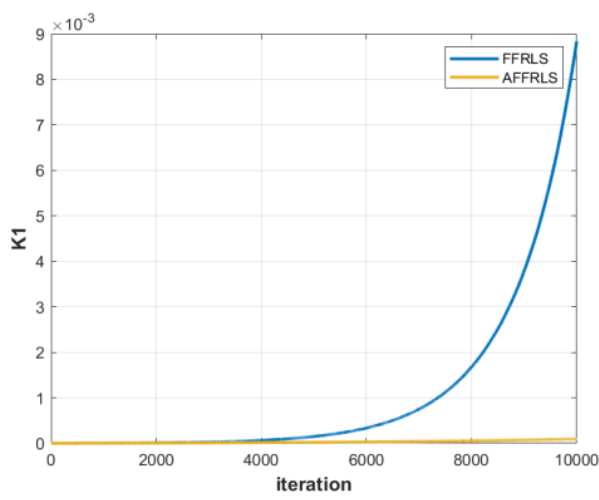

(b)

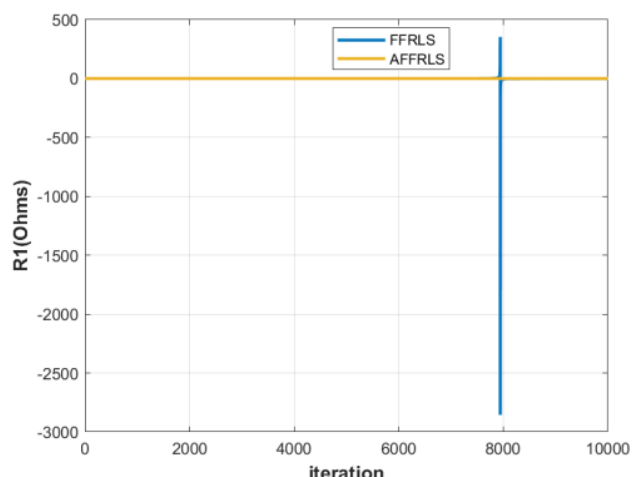

(d)

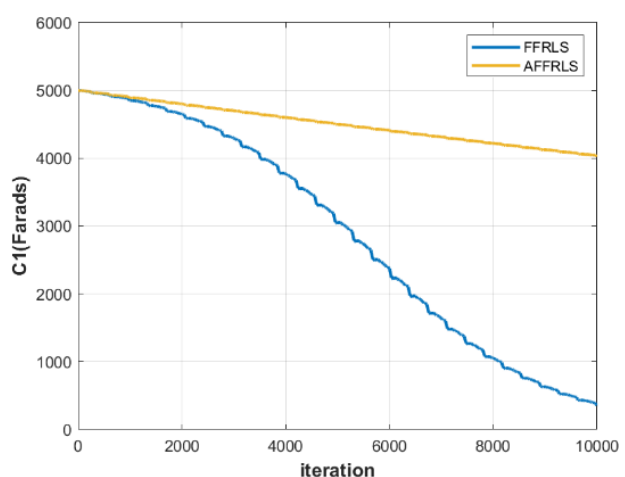

(f)

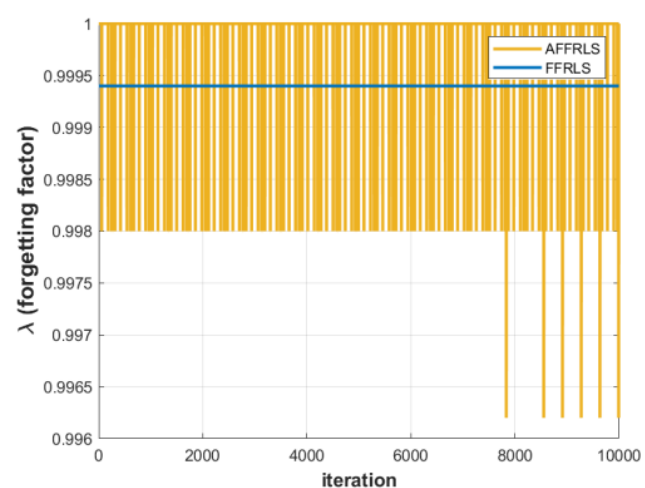

(h)

Figure. 6 Parameter identification results for FFRLS and AFFRLS algorithm: (a),(b),(c) Identification curve of Nernst parameters equation K0, K1 and K2 respectively, (d) Identification curve of R1, (e) Identification curve of R2, (f) Identification curve of $\mathrm{C} 1,(\mathrm{~g})$ Identification curve of $\mathrm{M}$, and (h) Identification curve of the forgetting factor $\lambda$ 


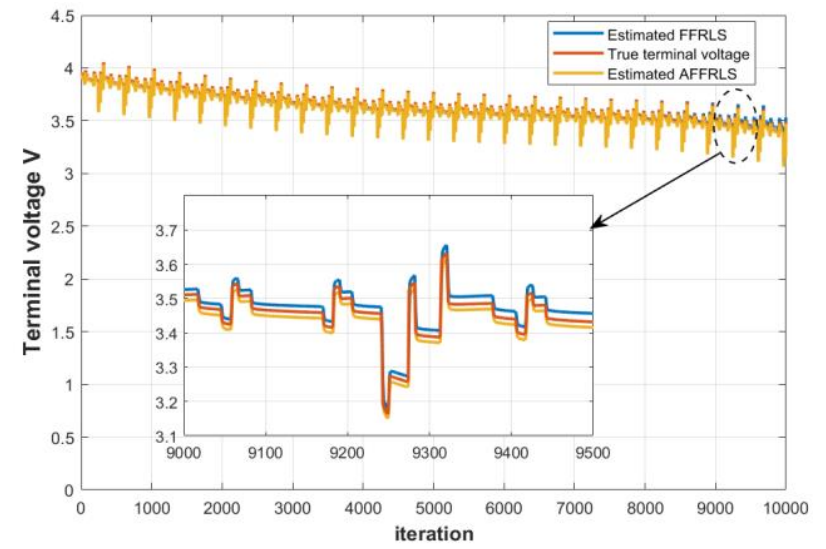

(a)

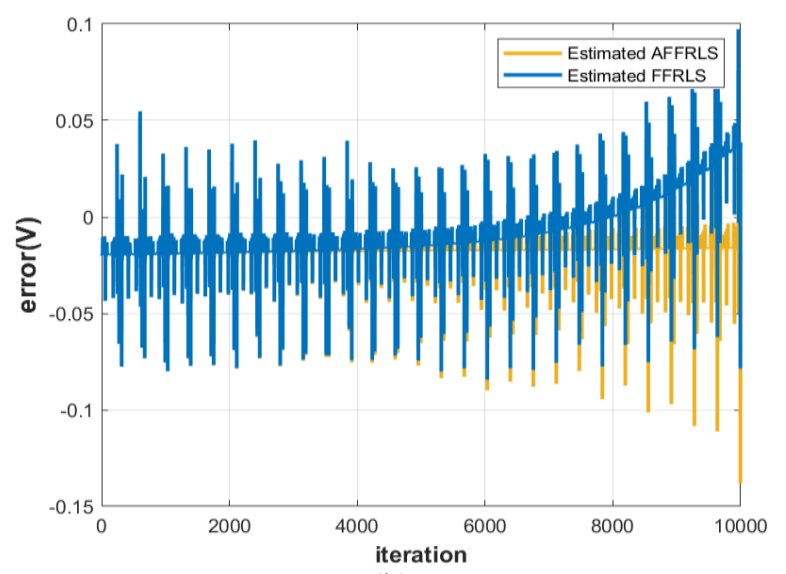

(b)

Figure. 7 (a) True terminal voltage vs terminal voltage estimated by FFRLS and AFFRLS algorithms and (b) Estimation error for the two algorithms

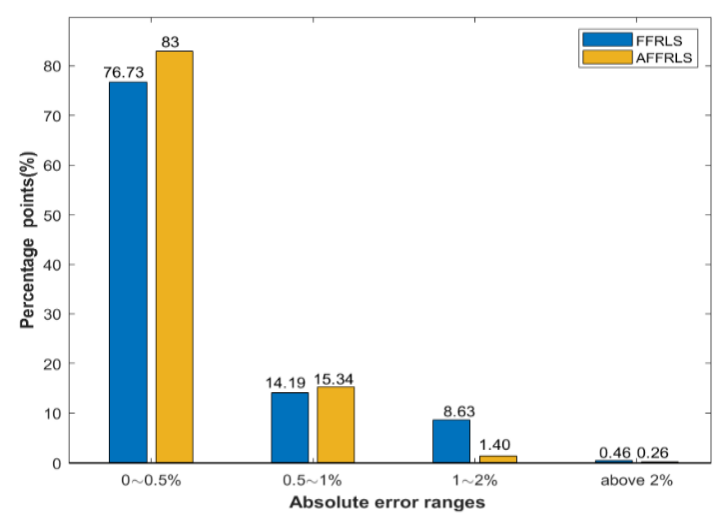

Figure. 8 Percentage of absolute relative error points for FFRLS and AFFRLS in DST

True Terminal voltage and estimated terminal voltage using FFRLS and AFFRLS are depicted in Fig. 7(a). The terminal voltage error for the two algorithms is shown in Fig. 7(b).

Both algorithms were very close from the true terminal voltage, although AFFRLS showed small voltage error compared with FFRLS. This can be well observed in Fig. 8 where we display the distribution statistics of absolute relative errors.
Fig. 8 is obtained by counting the points of absolute relative errors falling into each interval $(0 \sim 0.5 \%, 0.5 \sim 1 \% \ldots)$ and then express it in percentage.

As reflected from Fig.8, AFFRLS has a more accurate parameter identification ability than the original FFRLS algorithm. As can be seen in this figure, the percentage of absolute relative error distribution of AFFRLS for small errors is substantially higher than that of FFRLS: $83 \%$ for AFFRLS versus $76.73 \%$ for FFRLS in the scale of 0 to $0.5 \%$. $15.34 \%$ versus $14.19 \%$ for errors in the range of $(0.5-1 \%)$. The distribution lowers down for higher ranges: $8.63 \%$ for FFRLS against $1.4 \%$ for absolute relative errors between $1-2 \%$, and $0.46 \%$ for FFRLS over $0.26 \%$ for AFFRLS.The superiority of the new AFFRLS over the classic FFRLS will be much clearer in the FUDS test drive which is considered more aggressive than the DST dynamic profile.

\subsection{Federal urban driving schedule (FUDS)}

The current profile for this test can be visualised in Fig. 5. We can see that the current profile of FUDS is more unstable and has more oscillation than that of DST.

We proceeded in the same manner as we did in the DST case. We initialised both algorithms with the required parameters: $(\theta(0), G(0), P(0), U 1(0))$, and the forgetting factor $\lambda$.

After that the algorithms iterate (we run the algorithms separately in MATLAB) to estimate the battery parameters hold in $\theta(\mathrm{k}) \mathrm{Eq}$. (6) and then predict the terminal voltage $\mathrm{V}(\mathrm{k}) \mathrm{Eq}$. (3), in every iteration we fed the algorithms with the required parameters (current voltage, time.) stored in the dataset.Fig. 9 illustrates the parameter identification results of the FFRLS and AFFRLS for FUDS test.

As obtained for the first test, it can be seen that the parameters identified by the FFRLS algorithm have more fluctuations and more spikes but the identification ability is insufficient. This means that the algorithm doesn't accurately reflect the complex characteristics of real-time variation when battery is charged and discharged rapidly compared with AFFRLS. Fig. 11 demonstrate this observation where we can see that FFRLS has a high distribution for large error than AFFRLS

The parameters identified by the AFFRLS on the other side are more stable except the forgetting factor $\lambda$ which oscillate vigorously, due to the fact that current changes rapidly in this dynamic profile. Allowing $\lambda$ to change gives more flexibility and adaptability to identify parameters in vigorous tests such as FUDS. 


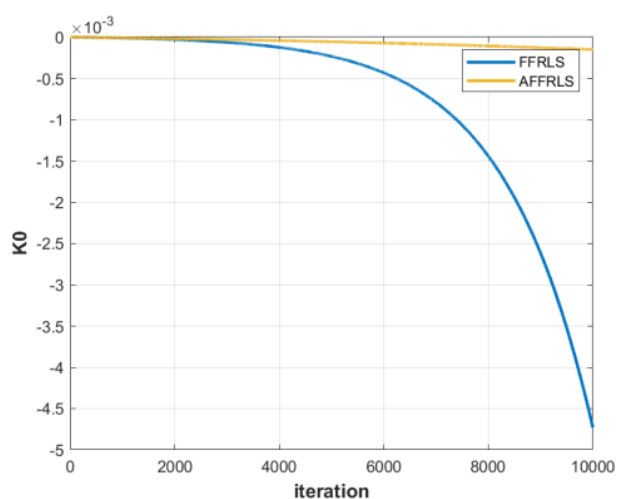

(a)

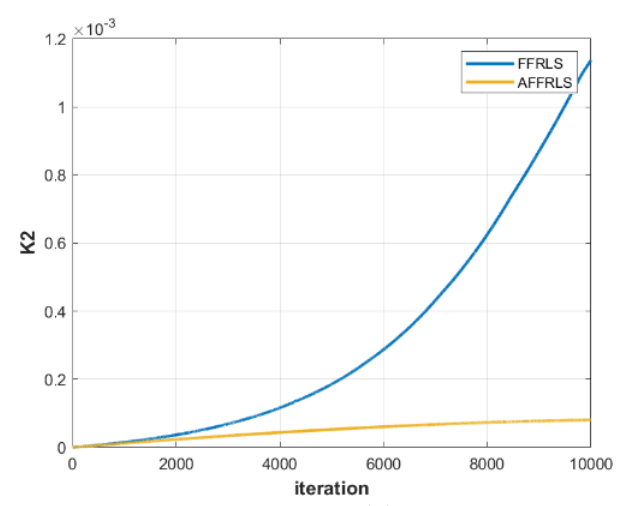

(c)

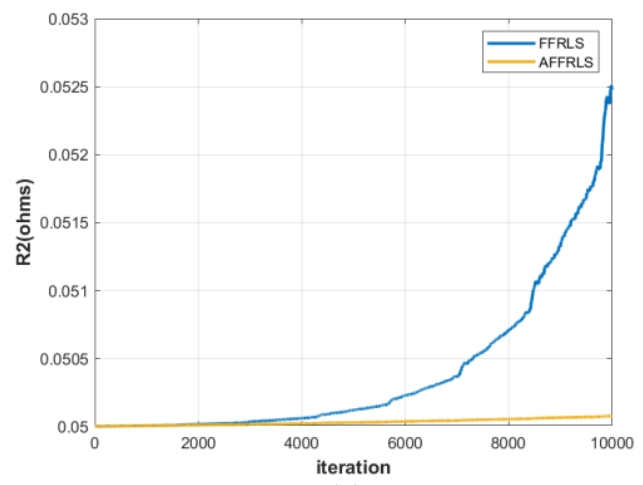

(e)

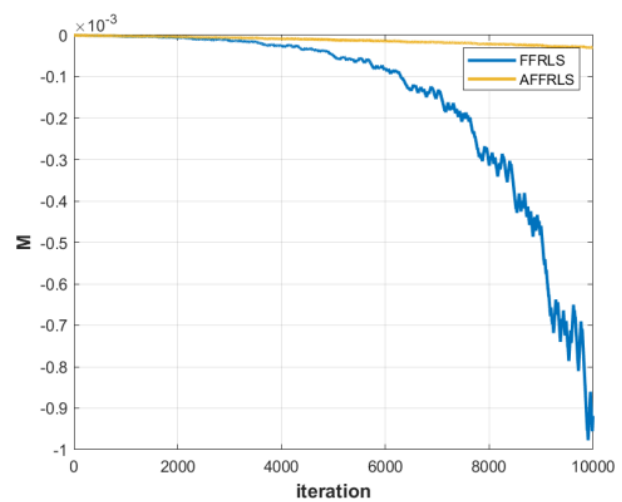

( $\mathrm{g})$

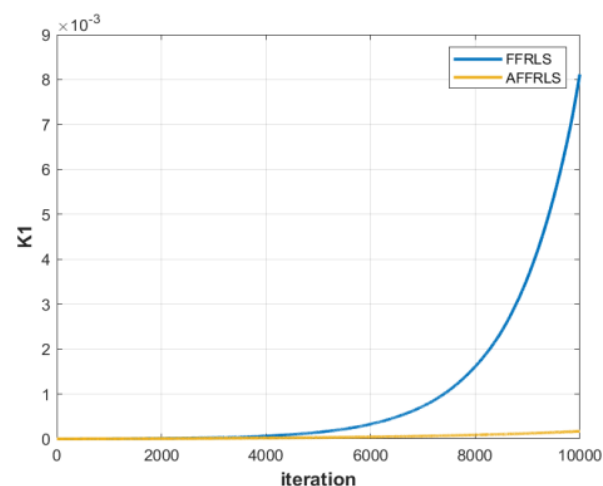

(b)

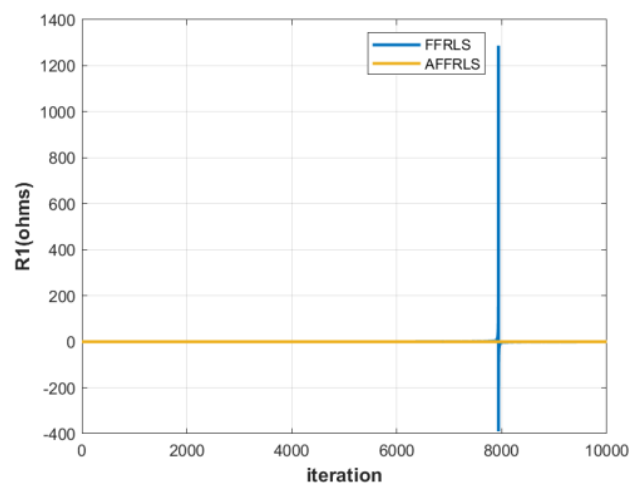

(d)

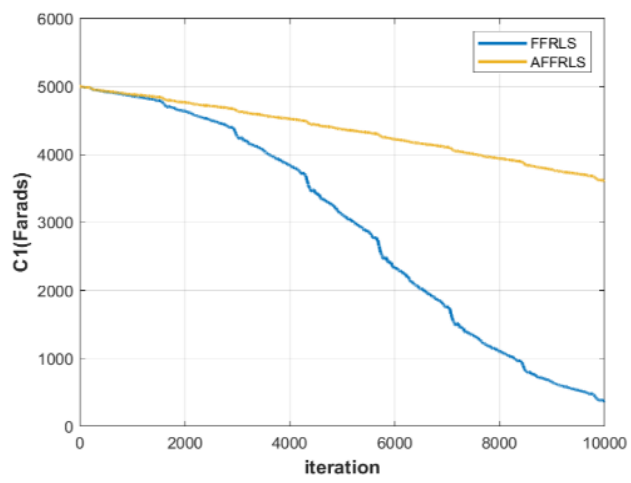

(f)

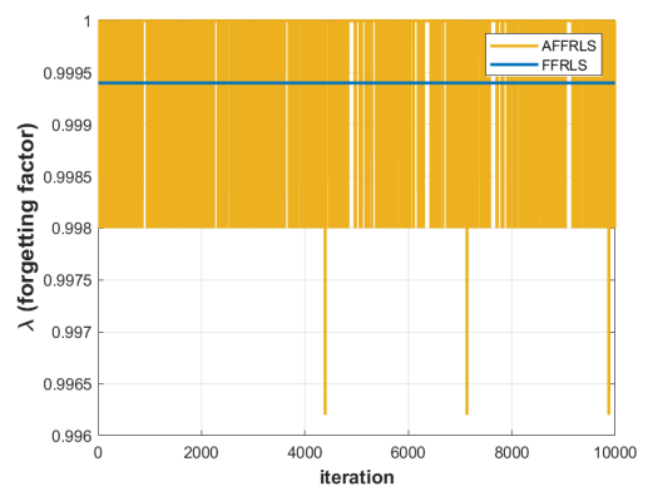

(h)

Figure. 9 Parameter identificatio1n results for FFRLS and AFFRLS algorithm: (a), (b), (c) Identification curve of Nernst parameters equation K0, K1 and K2 respectively, (d) Identification curve of R1, (e) Identification curve of R2, (f) Identification curve of $\mathrm{C} 1,(\mathrm{~g})$ Identification curve of M, and (h) Identification curve of Lambda $(\lambda)$ 


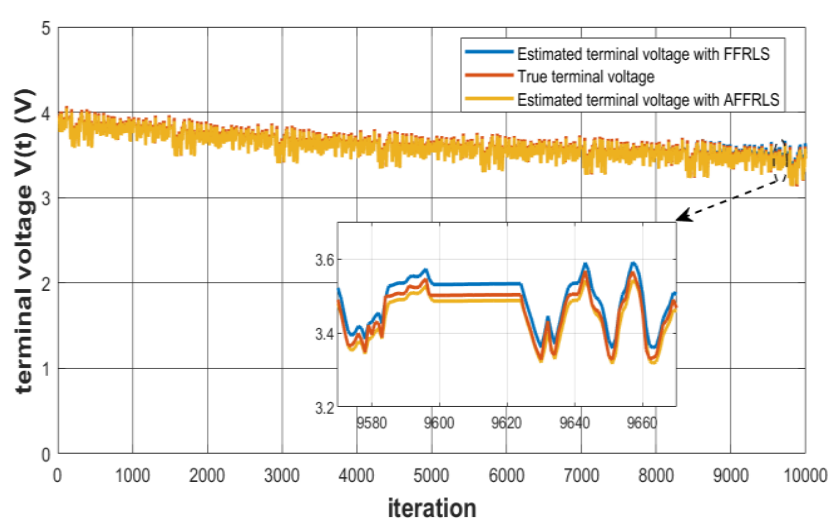

(a)

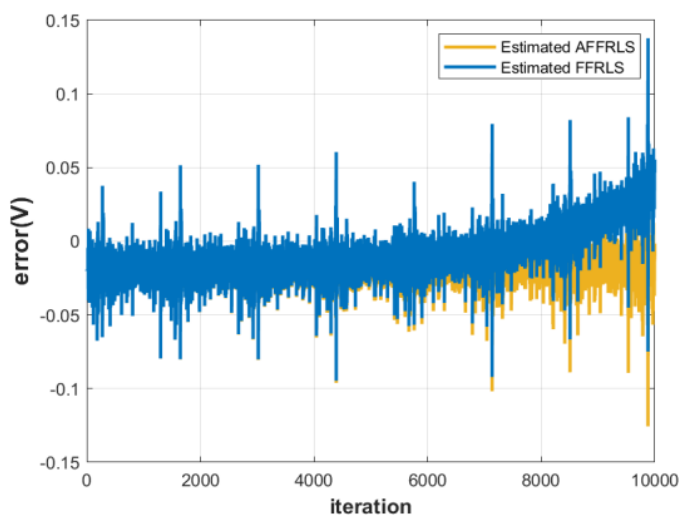

(b)

Figure.10 (a) True terminal voltage vs terminal voltage estimated by FFRLS and AFFRLS algorithms and (b)

Estimation error for the two algorithms

Fig. 10 shows the true terminal voltage and both identified voltage by FFRLS and AFFRLS, the estimated voltage error is plotted in the same figure.

From Fig. 10 we can observe that both algorithms are close from each other, but looking deeper we can see that AFFRLS is more accurate and precise Fig.11.

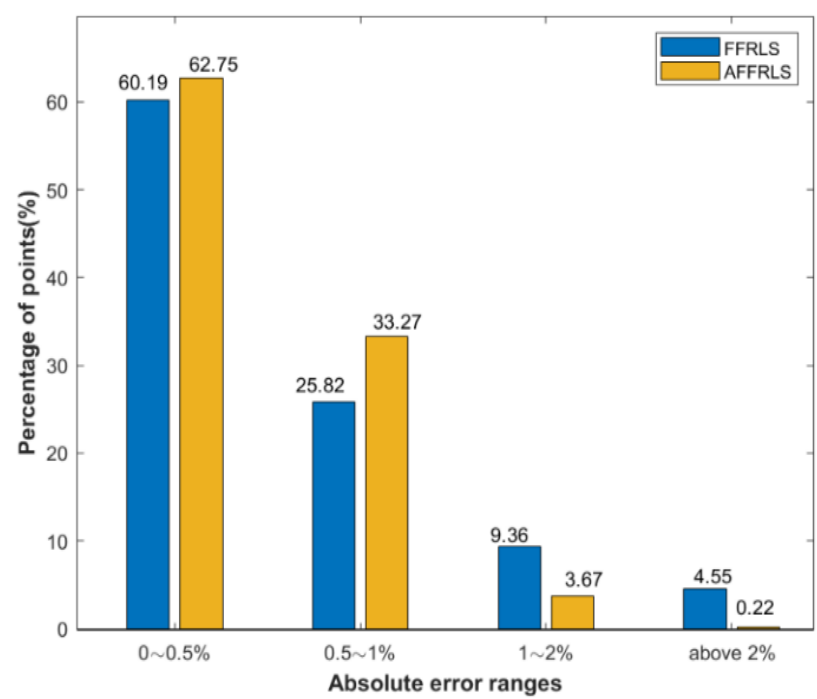

Figure. 11 Percentage of absolute relative error points for FFRLS and AFFRLS in FUDS
Fig. 11 is plotted in the same manner as Fig. 8 . We draw the same conclusion as for the DST test : AFFRLS algorithm is more precise than FFRLS in term of parameter identification ability. As we can observe in Fig. 11, AFFRLS has high distribution in small error range and low distribution for higher range compared to FFRLS in terms of absolute relative error: for absolute relative error between 0 and $0.5 \%$ AFFRLS scored $62.75 \%$ compared to $60.19 \%$ for FFRLS, $33.22 \%$ of the point in the FUDS test were between 0.5 and $1 \%$ for AFFRLS against $25.83 \%$ for FFRLS.As the relative error range goes up (the magnitude of the errors) the distribution lowers down for AFRRLS ,as we can see $3.67 \%$ of the errors were between 1 and $2 \%$ versus $9.36 \%$ for FFRLS and only $0.22 \%$ against $4.55 \%$ for errors above $2 \%$.

\section{Conclusion}

In this work, a parallel resistance-capacitance (RC) network is used as a model of lithium-ion battery for electric vehicle application,with OCV (open circuit voltage) SOC (state of charge) relationship discribed with an adaptation of Nernst equation.A zero state correction term is used to characterize the hysterisis phenomenon.

The identification of the equivalent circuit model parameters based on a proposed algorithm (AFFRLS) is studied and compared with the original FFRLS.

The estimated terminal voltage obtained by both algorithms (AFFRLS and FFRLS) is compared with the actual terminal voltage. The correctness of the equivalent circuit model parameter identification was confirmed using data collected from the center for advanced life cycle engineering (CALCE) battery group for two EV cycles named Dynamic Stress Test (DST) and Federal Urban Driving Schedule (FUDS).

We supplied the data and compared the estimated output voltage by the two algorithms (AFFRLS and FFRLS) in both dynamic tests (DST and FUDS).and showed that the absolute relative error distribution of AFFRLS in high range (relative error above 2\%) is low up to $4 \%$ less than FFRLS.This means AFFRLS is more superior over FFRLS for battery model parameter identification in term of accuracy.

\section{Conflicts of Interest}

The authors declare no conflict of interest.

\section{Author Contributions}

The main conceptual ideas, the methodology and software analysis were worked out by Mouncef Elmarghichi, and Mostafa bouzi.The validation parts, 
formal analysis, Investigation, resources, data Curation, were taken care by Mouncef Elmarghichi and Naoufl Ettalabi.For writing: the original draft preparation as well as for review editing were taking care by Mouncef Elmarghichi,Mostafa bouzi and Naoufl Ettalabi.The visualization, supervision and administration was taking care by Mouncef Elmarghichi and Naoufl Ettalabi. Funding acquisition was taken care by Mouncef Elmarghichi.

\section{References}

[1] M. A. Hannan, M. H. Lipu, A. Hussain, and A. Mohamed, "A review of lithium-ion battery state of charge estimation and management system in electric vehicle applications: Challenges and recommendations", Renewable and Sustainable Energy Reviews, Vol. 78, pp. 834-854, 2017.

[2] P. Shrivastava, T. K. Soon, M. Y. I. B. Idris, and S. Mekhilef, "Overview of model-based online state-of-charge estimation using Kalman filter family for lithium-ion batteries", Renewable and Sustainable Energy Reviews, Vol. 113, pp. 109233, 2019.

[3] D. W. Chung and S. H. Yang, "SOC estimation of lithium-ion battery based on kalman filter algorithm for energy storage system in microgrids", In: Proc. of E3S Web of Conferences, on Energy Storage Equipment Optimization and Analysis, Gwangju-city, South Korea, pp. 02006, 2018.

[4] M. Paschero, G. L. Storti, A. Rizzi, F. M. F. Mascioli, and G. Rizzoni, "A novel mechanical analogy-based battery model for SoC estimation using a multicell EKF", IEEE Transactions on Sustainable Energy, Vol. 7, No. 4, pp. 16951702, 2016.

[5] Z.L. Zhang, X. Cheng, Z.Y. Lu, and D.J. Gu, "SOC estimation of lithium-ion batteries with AEKF and wavelet transform matrix", IEEE Transactions on Power Electronics, Vol. 32, No. 10, pp. 7626-7634, 2016.

[6] B. Ning, B. Cao, B. Wang, and Z. Zou, "Adaptive sliding mode observers for lithiumion battery state estimation based on parameters identified online", Energy, Vol. 153, pp. 732742, 2018.

[7] Y. Feng, C. Xue, Q.-L. Han, F. Han, and J. Du, "Robust Estimation for State-of-Charge and State-of-Health of Lithium-ion Batteries Using Integral-Type Terminal Sliding-Mode Observers", IEEE Transactions on Industrial Electronics, Vol. 67, No. 5, pp. 4013 - 4023, 2019.
[8] N. Tian, Y. Wang, J. Chen, and H. Fang, "On parameter identification of an equivalent circuit model for lithium-ion batteries", In: Proc. of 2017 IEEE Conference ,on Control Technology and Applications (CCTA): IEEE, Hawai, USA, pp. 187-192, 2017.

[9] Z. Yu, L. Xiao, H. Li, X. Zhu, and R. Huai, "Model parameter identification for lithium batteries using the coevolutionary particle swarm optimization method", IEEE Transactions on Industrial Electronics, Vol. 64, No. 7, pp. 5690-5700, 2017.

[10] Z. Lao, B. Xia, W. Wang, W. Sun, Y. Lai, and M. Wang, "A novel method for lithium-ion battery online parameter identification based on variable forgetting factor recursive least squares", Energies, Vol. 11, No. 6, pp. 1358, 2018.

[11] J. Wang, L. Zhang, D. Xu, P. Zhang, and G. Zhang, "A simplified fractional order equivalent circuit model and adaptive online parameter identification method for lithium-ion batteries", Mathematical Problems in Engineering, Vol. 2019, 2019.

[12] Z. Yu, L. Xiao, H. Li, X. Zhu, and R. Huai, "Model parameter identification for lithium batteries using the coevolutionary particle swarm optimization method", IEEE Transactions on Industrial Electronics, Vol. 64, No. 7, pp. 5690-5700, 2017.

[13] W. Xu, J. Xu, and X. Yan, "Lithium-ion battery state of charge and parameters joint estimation using cubature Kalman filter and particle filter", Journal of Power Electronics, Vol. 20, No. 1, pp. 292-307, 2020.

[14] P. S. Diniz, Adaptive Filtering Algorithms and Practical Implementation, Vol. 694, Springer, Switzerland, 2020.

[15] X. Sun, J. Ji, B. Ren, C. Xie, and D. Yan, "Adaptive Forgetting Factor Recursive Least Square Algorithm for Online Identification of Equivalent Circuit Model Parameters of a Lithium-Ion Battery", Energies, Vol. 12, No. 12, pp. 2242, 2019.

[16] X. Hu, F. Sun, Y. Zou, and H. Peng, "Online estimation of an electric vehicle lithium-ion battery using recursive least squares with forgetting", In: Proc. of the American Control Conference, San Francisco, USA, pp. 935-940, 2011.

[17] Y. Tian, Z. Zeng, J. Tian, S. Zhou, and C. Hu, "Joint estimation of model parameters and SOC for lithium-ion batteries in wireless charging systems", In: Proc. of IEEE PELS Workshop on 
Emerging Technologies on Wireless Power Transfer, Chongqing, China, pp. 263-267, 2017.

[18] F. Zheng, Y. Xing, J. Jiang, B. Sun, J. Kim, and M. Pecht, "Influence of different open circuit voltage tests on state of charge online estimation for lithium-ion batteries", Applied Energy, Vol. 183, pp. 513-525, 2016.

[19] Y. Xing, W. He, M. Pecht, and K. L. Tsui, "State of charge estimation of lithium-ion batteries using the open-circuit voltage at various ambient temperatures", Applied Energy, Vol. 113, pp. 106-115, 2014.

[20] W. He, N. Williard, C. Chen, and M. Pecht, "State of charge estimation for Li-ion batteries using neural network modeling and unscented Kalman filter-based error cancellation", International Journal of Electrical Power \& Energy Systems, Vol. 62, pp. 783-791, 2014. 